TITLE:

\title{
Magnifying narrowband imaging is more accurate than conventional white-light imaging in diagnosis of gastric mucosal cancer.
}

\section{$\operatorname{AUTHOR}(\mathrm{S}):$}

Ezoe, Yasumasa; Muto, Manabu; Uedo, Noriya; Doyama, Hisashi; Yao, Kenshi; Oda, Ichiro; Kaneko, Kazuhiro; ...

Takeuchi, Yoji; Kaneko, Yoshibumi; Saito, Yutaka

\section{CITATION:}

Ezoe, Yasumasa ... [et al]. Magnifying narrowband imaging is more accurate than conventional white-light imaging in diagnosis of gastric mucosal cancer.. Gastroenterology 2011, 141(6): 2017-2025.e3

\section{ISSUE DATE:}

2011-12

URL:

http://hdl.handle.net/2433/151866

\section{RIGHT:}

(C) 2011 AGA Institute. Published by Elsevier Inc.; この論文は出版社版で ありません。引用の際には出版社版をご確認ご利用ください。; This is not the published version. Please cite only the published version. 


\title{
Magnifying Narrowband Imaging Versus Conventional White-Light Imaging for Accurate Diagnosis of Gastric Mucosal Cancer
}

\section{Short Title:}

Magnifying NBI for diagnosis of small gastric cancer

\begin{abstract}
Authors:
Yasumasa Ezoe, Manabu Muto, Noriya Uedo, Hisashi Doyama, Kenshi Yao, Ichiro Oda, Kazuhiro Kaneko, Yoshiro Kawahara, Chizu Yokoi, Yasushi Sugiura, Hideki Ishikawa, Yoji Takeuchi, Yoshibumi Kaneko, and Yutaka Saito.
\end{abstract}

\section{Author Affiliations:}

Kyoto University, 54 Kawahara-cho, Shogoin, Sakyo-ku, Kyoto, 606-8507 Japan (Y Ezoe, M Muto)

Osaka Medical Cancer for Cancer and Cardiovascular Diseases, 1-3-3 Nakamichi, Higashinari-ku, Osaka, 537-8511 Japan (N Uedo, Y Takeuchi)

Ishikawa Prefectural Central Hospital, 2-1 Kuratsukihigashi, Kanazawa, Ishikawa, 920-8530 Japan (H Doyama, Y Kaneko)

Fukuoka University Chikushi Hospital, 1-1-1 Zokumyo, Chikushino, Fukuoka, 818-8502 Japan (K Yao)

National Cancer Center Hospital, 5-1-1 Tsukiji, Chuo-ku, Tokyo, 104-0045 Japan (I Oda, Y Saito)

National Cancer Center Hospital East, 6-5-1 Kashiwanoha, Kashiwa-shi, Chiba, 277-8577 Japan (K Kaneko) 
Okayama University, 2-5-1 Shikata-cho, Okayama-shi, Okayama, 700-8558 Japan (Y Kawahara)

National Center for Global Health and Medicine, 1-21-1 Toyama, Shinjuku-ku, Tokyo, 1628655 Japan (C Yokoi)

Kitano Hospital, 2-4-20 Ohgimachi, Kita-ku, Osaka, 530-8480 Japan (Y Sugiura)

Kyoto Prefectural University of Medicine, 465 Kajii-cho, Hirokoji Agaru, Kawaramachi-dori, Kamigyo-ku, Kyoto, 602-8566 Japan (H Ishikawa)

\section{Grant Support:}

This study was supported by a part of grant-in-aid for cancer research from the Ministry of Health (H21-009), Labor, and Welfare of Japan.

\section{Abbreviations:}

CI, confidence interval; C-WLI, conventional white-light imaging; DL, demarcation line; EMR, endoscopic mucosal resection; ESD, endoscopic submucosal dissection; IM, irregular margin; IMVP, irregular microvascular pattern; IQR, interquartile range; M-NBI, magnifying narrowband imaging; NPV, negative predictive value; PPV, positive predictive value; SDA, spiny depressed area; STARD, Standards for the Reporting of Diagnostic Accuracy Studies.

\section{Corresponding Author:}

Manabu Muto

54 Kawahara-cho, Shogoin, Sakyo-ku, Kyoto 606-8507, Japan

e-mail:mmuto@kuhp.kyoto-u.ac.jp

telephone: $+81-75-751-4592$

Fax: +81-75-751-4303 


\section{Disclosures:}

We declare that we have no conflicts of interest.

\section{Author Contributions:}

YE, MM, KY, and HI were responsible for the conception and design of this study. YE, MM, NU, HD, KY, IO, KK, YK, CY, YS, YT, YK, and YS collected and assembled data. HI was responsible for data management. YE and MM analyzed and interpreted the data. All authors reviewed the data analyses and interpretations. YE and MM wrote the manuscript, which has been approved by all authors. 
BACKGROUND \& AIMS: Gastric cancer is the second leading cause of cancer death worldwide. Early diagnosis of the stage of mucosal cancer is one of the best strategies for improving survival. However, an accurate diagnosis of depressed gastric mucosal cancer is difficult using conventional white-light imaging (C-WLI) endoscopy. We aimed to compare the real-time diagnostic yield of C-WLI for small depressed gastric mucosal cancers with that of magnifying narrowband imaging (M-NBI). METHODS: We recruited a gastric-cancerenriched population for this multicenter, prospective, randomized controlled trial. Patients with newly detected, undiagnosed, depressed lesions $\leq 10 \mathrm{~mm}$ in diameter were randomly assigned in a 1:1 ratio to C-WLI or M-NBI immediately after detection. The C-WLI group underwent M-NBI after C-WLI. We compared the diagnostic accuracy, sensitivity, and specificity between C-WLI and M-NBI, and assessed the diagnostic yield of M-NBI conducted in conjunction with C-WLI. RESULTS: We screened 1353 patients using esophagogastroduodenoscopy. Of these, 362 with undiagnosed small depressed lesions were randomly assigned to M-NBI $(\mathrm{n}=182)$ or C-WLI $(\mathrm{n}=180)$; 353 were included in the analysis ( $\mathrm{n}=177$ and 176 , respectively). Forty gastric cancers (20 per group) were newly diagnosed. The diagnostic capabilities of M-NBI and C-WLI were (median): accuracy, 90.4\% and $64.8 \%$; sensitivity, $60.0 \%$ and $40.0 \%$; and specificity, $94.3 \%$ and $67.9 \%$, respectively. The accuracy and specificity of M-NBI were greater than those of C-WLI $(P<.001)$; the difference in sensitivity was not significant $(P=.34)$. M-NBI in conjunction with C-WLI significantly enhanced the performance of C-WLI alone: accuracy increased from (median) $40.0 \%$ to $95.0 \%, P<.001$; sensitivity increased from $67.9 \%$ to $96.8 \%, P<.001$; and specificity increased from $64.8 \%$ to $96.6 \%, P<.001$. CONCLUSION: M-NBI might represent a new standard examination modality for diagnosing depressed small gastric mucosal cancers accurately (Clinical trial number, UMIN-CTR000001072). Keywords: Gastric cancer; Early detection; Accuracy; NBI. 
Gastric cancer is the fourth most common malignancy and the second leading cause of death from cancer worldwide. ${ }^{1}$ Early detection and curative treatment are the best strategies for improving patient survival. Esophagogastroduodenoscopy is the most sensitive method of early detection of gastric cancers. However, an accurate early diagnosis of gastric mucosal cancer is difficult with conventional white-light imaging (C-WLI) endoscopy; nevertheless, it remains the standard endoscopic examination modality worldwide.

Detection of mucosal cancers $\leq 20 \mathrm{~mm}$ in diameter is ideal, as they are curable using minimally invasive treatments such as endoscopic mucosal resection (EMR) and endoscopic submucosal dissection (ESD). ${ }^{2,3}$ Among the gastric mucosal cancers, the depressed type is the predominant morphology. ${ }^{4-6}$ However, small depressed cancers $(\leq 10 \mathrm{~mm}$ in diameter) are more difficult to distinguish from benign abnormalities (such as inflammation) compared with elevated cancers. Although chromoendoscopy using indigo carmine has contributed to an improvement in the diagnosis of gastric mucosal cancers, ${ }^{7}$ there is no evidence of the superiority of chromoendoscopy over C-WLI. Therefore, C-WLI endoscopy remains the standard imaging modality for diagnosing gastric mucosal cancers.

Histological evaluation of biopsy specimens from suspicious lesions is conventionally used to confirm a diagnosis. A highly accurate diagnosis without the need for a biopsy is the ultimate goal of endoscopists, as this would decrease the number of unnecessary biopsies, especially when confirming a negative biopsy of any suspicious cancerous lesion. This could reduce the risk of postbiopsy bleeding, costs associated with the procedure, and the workload on pathologists.

Magnifying narrowband imaging (M-NBI), a recently developed advanced endoscopic imaging technology, was reported to be useful for the accurate diagnosis of gastric abnormalities such as cancers, ${ }^{8-13}$ adenomas, ${ }^{14}$ and intestinal metaplasia. ${ }^{15}$ However, no randomized trials have been conducted to compare M-NBI with C-WLI. The present study 
was designed to assess and compare the real-time diagnostic yield of C-WLI for depressed gastric mucosal cancers with that of M-NBI when performed by skilled endoscopists.

\section{Methods}

\section{Study Design and Participants}

This randomized, controlled, open-label, multicenter trial was conducted at nine centers in Japan. This study was conducted according to the Standards for the Reporting of Diagnostic Accuracy Studies (STARD) initiative ${ }^{16}$ and the Declaration of Helsinki.

The frequency of synchronous or metachronous multiple gastric cancers was reported as 3-5 per 100 patient years, ${ }^{17-19}$ which is higher than the incidence of gastric cancer in the general population. In other words, patients with gastric cancer might constitute a cancerenriched population, which may be a more suitable model for screening of potential gastric cancers than the general population. Therefore, we recruited patients aged 20 years or older with untreated gastric cancers and patients with a history of gastric cancer. Patients who had been treated with EMR or ESD were included in the latter group, as their stomachs were preserved with minimum injury. We excluded patients who had been treated with surgical resection, as the stomach was either removed or was reduced in size. Other exclusion criteria were serious complications that could interfere with the examination protocol and the use of medication that might interfere with the collection of a biopsy specimen. Written informed consent was obtained, and the institutional review board of each participating hospital approved the study. The clinical trial number of this study was UMIN-CTR000001072.

To detect a target lesion, screening was done using C-WLI endoscopy. Previously undetected lesions were considered ideal potential targets for evaluating the diagnostic yield without bias. Therefore, the target lesions for this study were "newly detected and undiagnosed" small depressed gastric lesions $\leq 10 \mathrm{~mm}$ in diameter. We did not target lesions 
that had been analyzed histologically. Small depressed lesions with apparent erosion or ulceration were also not evaluated, as it is difficult to visualize surface changes in these lesions. If the patient had multiple such lesions, only the first lesion detected was selected for examination. The diameter of each lesion was estimated by comparing it with the size of the biopsy forceps.

\section{Randomization and Masking}

When a target small depressed lesion was detected by C-WLI screening, patients were immediately assigned randomly to undergo detailed examination using C-WLI or M-NBI at a 1:1 ratio. After the randomization, all endoscopists knew which imaging method would be used for the detailed examination when making a diagnosis of the target lesion.

Randomization was performed promptly on-site using tables of random numbers stratified by hospital, and the results thereof were kept in sealed, numbered envelopes. The random allocation sequence was prepared at the data management center. Both the assignment result and the corresponding envelope number were recorded by the data management center. At each participating hospital, sealed envelopes were stored by a third party who was not involved in the study, and the envelopes were opened by an assistant physician in serial order only when randomization was performed. The assigned patient identification number, envelope number, and the assignment result were recorded on-site and faxed to the data management center on the day of the examination.

\section{Procedure and Endpoints}

The study design and the protocol examination were outlined in the supplemental figure

(Supplement 1). The diagnosis for the target lesion was made by one endoscopist according to predetermined diagnostic criteria for C-WLI and M-NBI without any consultation with other physicians, and an assistant physician immediately recorded the results using a case 
report form. For each modality, the interval between the start of the observation and the time at which an endoscopic diagnosis was made was measured using a stopwatch. For the C-WLI group, M-NBI examination was performed after completion of a diagnosis based on C-WLI. This procedure was used to evaluate the effect of using M-NBI in conjunction with C-WLI. After all records were compiled, at least one biopsy specimen was obtained from the target lesion.

The primary aim of the study was to compare the diagnostic accuracy between C-WLI and M-NBI. The secondary aim was to compare diagnostic sensitivity, specificity, and examination time between C-WLI and M-NBI, and to evaluate the effects of an additional MNBI study after the initial C-WLI in terms of diagnostic accuracy, sensitivity, specificity, and examination time. Histopathology diagnosis of obtained biopsy specimens was used as a gold $\underline{\text { standard for the diagnosis. }}$

\section{Endoscopy System}

The NBI system is an innovative optical-image-enhanced technology that involves a narrow bandwidth NBI filter and is mounted in the video endoscopy system. The central wavelengths of the NBI filters are $415 \mathrm{~nm}$ and $540 \mathrm{~nm}$, and each has a bandwidth of $30 \mathrm{~nm}$. As $415 \mathrm{~nm}$ and $540 \mathrm{~nm}$ light are well absorbed by hemoglobin, the microvascular architecture of the mucosal surface can be visualized readily. Details of this system have been published elsewhere. $^{20-22}$

We used high-resolution magnifying endoscopy with a capability of 80-fold optical magnification (GIF-Q240Z, GIF-H260Z, and GIF-FQ260Z; Olympus Medical Systems, Tokyo, Japan) and a high-resolution liquid-crystal monitor (OEV191H; Olympus Medical Systems). We alternated between the two imaging modalities (C-WLI and M-NBI) by pushing a button on the endoscope (EVIS LUCERA SPECTRUM system, Olympus Medical 
Systems). We used a fixed-structure-enhancement setting and color tone for the video processor.

\section{Participating Endoscopists}

All examinations were performed by 31 endoscopic specialists accredited by the Japan Gastroenterological Endoscopy Society in nine institutes. Before the onset of the study, all participating endoscopists were trained using images of small depressed lesions to minimize diagnostic variation between them.

\section{Diagnostic Criteria for C-WLI and M-NBI}

Figure 1 shows a representative endoscopic image of a small depressed gastric cancer and a small depressed benign lesion. The endoscopic diagnostic criteria for small depressed gastric cancers using C-WLI were defined based on previous reports of C-WLI findings: an irregular margin (IM) and a spiny, depressed area (SDA). ${ }^{23}$ The observation of two findings (IM and SDA) in the target lesion was classified according to three categories: present, absent, or indeterminate.

The endoscopic diagnostic criteria for small depressed gastric cancers using M-NBI were defined based on previous reports by Yao et al.: a demarcation line (DL) between the depressed cancerous lesion and the surrounding noncancerous area and an irregular microvascular pattern (IMVP) inside the lesion. ${ }^{24}$ Observations of two findings (DL and IMVP) in the target lesion were also classified according to three categories: present, absent, or indeterminate.

Endoscopic diagnoses were determined according to the combined visibility of the two findings as follows (Supplement 2).

(1) If both findings were present, the diagnosis was "cancer."

(2) If either finding was indeterminate, the diagnosis was "inconclusive." 
(3) If either or both findings were absent, the diagnosis was "noncancerous."

For analyzing diagnostic accuracy, sensitivity, and specificity, lesions diagnosed as "inconclusive" were considered as endoscopic "noncancerous" lesions.

\section{Pathology Diagnosis}

The biopsy specimens were evaluated using hematoxylin-eosin staining. The diagnostic pathology criteria were based on the revised Vienna classification. ${ }^{25} \mathrm{C} 4$ (mucosal high-grade neoplasia) or C5 (submucosal invasion by neoplasia) were diagnosed as cancer, and C1 (negative for neoplasia), C2 (indefinite for neoplasia), or C3 (mucosal low-grade neoplasia) were diagnosed as noncancerous lesions. In this study, we used a central system of $\underline{\text { consultation with a main expert pathologist. If an indeterminate lesion were to be encountered, }}$ it was scheduled to be reviewed by this consulting pathologist in making a final diagnosis.

\section{Statistical Analysis}

We assumed that the accuracy, sensitivity, and specificity of C-WLI and M-NBI compared with histological diagnosis would be $60 \%$ and $85 \%$, respectively. To set a probability for error of 0.05 and attain a power of $80 \%$ for testing the superiority of M-NBI, 108 patients including at least 43 cancerous lesions were needed. Next, we calculated how many patients would need to be screened. As the frequency of small depressed lesions was reported to be $8.1 \%$ in the general population, ${ }^{9}$ the required size of the screening sample was 1100 patients.

Statistical analysis was performed using SPSS software, version 17 (SPSS Inc., Chicago, IL, USA). Diagnostic performance: accuracy, sensitivity, specificity, positive predictive value (PPV), and negative predictive value (NPV) are presented as percentages with $95 \%$ confidence intervals (CIs). Continuous variables are expressed as medians and interquartile ranges (IQRs). Analyses of the difference in diagnostic performance between C- 
WLI and M-NBI were conducted using the population whose diagnoses had been confirmed by pathology using Pearson's Chi-squared test. Analyses of the effect of additional M-NBI after the initial C-WLI on diagnostic performance were conducted using the population whose diagnoses had been confirmed by pathology and McNemar testing. Analysis of the examination duration was conducted using the population who completed protocol examination and the Mann-Whitney nonparametric test for comparisons between C-WLI and M-NBI, and the Wilcoxon signed-rank test for comparisons between C-WLI and C-WLI + M-NBI. All probability values calculated in this analysis were 2 -sided, and $P<.05$ was considered significant.

\section{Results}

Between June 2008 and May 2010, 1365 patients were enrolled in the study. Eight patients refused to participate and 4 were registered twice; therefore, the remaining 1353 patients were registered correctly and underwent endoscopic screening. Screening was discontinued for 2 patients because of a large amount of residual digesta in the stomach and a severe vomiting reflex. Endoscopic screening was completed for the remaining 1351 patients.

Of the screened patients, $362(26.8 \%)$ had newly detected, undiagnosed small depressed lesions and were randomly assigned to one of two groups: (1) 180 patients were examined using C-WLI, followed by M-NBI; (2) 182 patients were examined using M-NBI alone. Four patients in the C-WLI group (1 patient's lesion was $>10 \mathrm{~mm}$ in diameter, 1 was discontinued from the examination because of Mallory-Weiss syndrome, and 2 had a missed biopsy) and 5 patients in the M-NBI group (1 was examined with an unpermitted endoscope and 4 missed biopsy) were excluded. Data for 176 patients of the C-WLI group and 177 patients of the M-NBI group were used for the final analysis (Figure 2). The demographic 
and lesion characteristics of the 2 groups were balanced. In both groups, $13 \%$ of patients had newly diagnosed gastric cancer (20 per group; Table 1).

Table 2 shows endoscopic diagnoses for all lesions. Inconclusive diagnoses were obtained for 3 lesions (1.7\%) using M-NBI, for 6 lesions (3.4\%) using C-WLI, and for 2 lesions (1.3\%) using C-WLI followed by M-NBI. These lesions were considered endoscopic "noncancerous" lesions for analysis.

The real-time diagnostic accuracy of M-NBI was significantly greater than that of CWLI (90.4\% [95\% CI, 85.1-94.3\%] and 64.8\% [95\% CI, 57.2-71.8\%], respectively; $P$ $<.001$; Table 3). Real-time M-NBI diagnosis had greater specificity than C-WLI diagnosis (94.3\% [95\% CI, 89.4-97.3\%] and 67.9\% [95\% CI, 60.0-75.2\%], respectively; $P<.001$; Table 3). The diagnostic sensitivities of M-NBI and C-WLI did not differ significantly (60.0\% [95\% CI, 36.1-80.9\%] and 40.0\% [95\% CI, 19.1-63.9\%], respectively; $P=.34$; Table 3). M-NBI in conjunction with C-WLI significantly enhanced the diagnostic performance of the latter: accuracy increased from $67.9 \%$ (95\% CI, 60.0-75.2\%) to $96.8 \%(92.7-99.0 \% ; P$ $<.001)$; sensitivity increased from $40.0 \%$ (95\% CI, 19.1-63.9\%) to 95.0\% (75.1-99.9\%; $P$ $<.001$ ); and specificity increased from $64.8 \%$ (95\% CI, 57.2-71.8\%) to 96.6\% (95\% CI, 93.5-99.1\%; $P<.001$; Table 3).

The median durations of the C-WLI and M-NBI procedures were $21 \mathrm{~s}$ (IQR, 12-40 s) and $55 \mathrm{~s}(\mathrm{IQR}, 23-97 \mathrm{~s})$, respectively, and this difference was highly significant $(P<.001)$. The median total duration of C-WLI followed by M-NBI (72 s [IQR, 40-144 s]) was significantly longer than that of C-WLI alone $(P<.001)$. All patients tolerated the procedures well (Table 3).

Figure 3 shows the PPV and NPV data for each examination. M-NBI significantly improved the PPV compared with C-WLI alone to $57.1 \%$ (95\% CI, 36.0-78.3\%) from 13.8\% (95\% CI, 2.9-22.7\%; $P=.001)$. Furthermore, C-WLI followed by M-NBI dramatically 
improved the PPV from $13.8 \%$ (95\% CI, 2.9-22.7\%) to 79.2\% (95\% CI, 62.9-95.4; $P<.001)$. Similarly, the NPV of C-WLI of $89.8 \%$ (95\% CI, 84.4-95.3) was improved by M-NBI to 94.9\% (95\% CI, 91.4-98.3; $P=.16$ ) and by C-WLI followed by M-NBI to 99.3\% (95\% CI, $98.1-100 ; P<.001)$.

Detailed C-WLI examination was discontinued during the procedure in 1 patient $(1 / 362 ; 0.3 \%)$ because of bleeding associated with Mallory-Weiss syndrome. Although the bleeding stopped spontaneously without any endoscopic hemostatic treatment, a biopsy specimen was not obtained because the suspicious target lesion was missed. Two patients $(2 / 362 ; 0.6 \%)$ were hospitalized on the day after examination because of bleeding from the biopsy site; although 1 patient needed a blood transfusion, both patients were discharged within a few days. None of the 3 patients experienced prolonged adverse effects. There were no serious adverse events directly related to the endoscopic observations.

Table 4 summarizes the clinical courses and pathological diagnoses of 40 gastric cancers in 40 patients. Thirty-two patients were treated endoscopically (by EMR or ESD). Five patients underwent surgical resection for synchronous advanced gastric cancers. The remaining 3 patients did not receive any treatment; 2 had other concomitant noncurable malignancies, and 1 refused treatment. Histologically, 39 lesions were of the intestinal type and one lesion was of the diffuse type. Regarding the depth of the 37 lesions that were removed, 35 were mucosal cancers, 2 of which were accompanied by submucosal invasion (0.3 $\mathrm{mm}$ and $0.8 \mathrm{~mm})$. The depths of the 3 untreated lesions were estimated endoscopically as 2 mucosal cancers and 1 submucosal cancer.

\section{Discussion}

In this multicenter, randomized trial, we compared the diagnostic yield of C-WLI with that of M-NBI for small gastric cancers. The primary aim of this study was to compare 
directly the real-time diagnostic accuracy of two randomly assigned endoscopic modalities.

One was the worldwide standard method of C-WLI; the other was M-NBI, which is the most advanced imaging method at present. This endpoint is the most important aspect of this study, because if C-WLI proves superior to M-NBI, such advanced methods are not needed in practice. However, if M-NBI is indeed better than C-WLI, it should be used more in daily practice. The secondary aim of this study was to evaluate the additional effect of performing $\underline{\text { M-NBI after C-WLI. This endpoint is also important, because in daily practice M-NBI are }}$ usually performed after carrying out C-WLI. Therefore, the results might reflect the practical diagnostic potential. To evaluate these aims, we used a strictly controlled randomized study. Furthermore, the endoscopic diagnosis in each method (C-WLI and M-NBI) was made on$\underline{\text { site and independently to avoid any bias. }}$

M-NBI, especially when used in conjunction with C-WLI, significantly enhanced realtime sensitivity, specificity, and accuracy of diagnosis, therefore, we concluded that M-NBI is essential modality for diagnosing small gastric mucosal cancer. Although there are reports on the diagnostic yield of M-NBI for differential diagnosis of gastric lesions, some were performed at only one institute,,${ }^{9,10,12,13}$ one was evaluated by several expert endoscopists using stored images and did not involve real-time assessment, ${ }^{12}$ and one included gastric lesions with a definite diagnosis. ${ }^{13}$ To overcome these limitations, our study targeted newly detected and undiagnosed gastric superficial lesions, which were evaluated on-site. For these reasons, the present results are the most reliable and could be a milestone in the field of endoscopic diagnosis of early gastric cancers.

Regarding accuracy and specificity, M-NBI alone yielded excellent results (90.4\% and 94.3\%, respectively), which were significantly better than those obtained with C-WLI. However, the sensitivities of M-NBI alone (60.0\%) and C-WLI alone (40.0\%) were lower than the estimated values: $85 \%$ for M-NBI and $60 \%$ for C-WLI. The low sensitivity of C- 
WLI might be acceptable considering the difficulty of diagnosing small gastric cancers in daily clinical practice. Although the reason for the low sensitivity of the M-NBI group is unknown, it might be associated with the examination protocol in this study; M-NBI observation was performed without evaluating a gross finding of lesions using C-WLI. In daily practice, magnifying examinations are usually performed after C-WLI. Actually, when performed after the C-WLI observation, M-NBI yielded excellent diagnostic performance in terms of accuracy, sensitivity, and specificity (all values were $>95 \%$ ). In addition, M-NBI and C-WLI followed by M-NBI significantly improved the PPV and NPV compared with C-WLI alone. This has enormous significance in clinical practice, because the examination with high PPV and high NPV might enable the clinician to make appropriate judgments as to which lesion needs pathology to confirm the diagnosis. When the lesion is suspected to be a neoplasm by C-WLI followed by M-NBI, taking a biopsy is highly recommended to confirm the pathology. On the other hand, when the lesion is not suspected to be a neoplasm by MNBI alone or by C-WLI followed by M-NBI, we could avoid a negative biopsy. These results have the potential to enable so-called "optic biopsy". Taken together, C-WLI followed by MNBI might be the best approach for making accurate diagnoses of small gastric cancers.

The durations of the M-NBI and C-WLI followed by M-NBI examinations were $34 \mathrm{~s}$ and $51 \mathrm{~s}$, respectively, significantly longer than that required for C-WLI alone. However, these durations are clinically acceptable, as we managed to make accurate diagnoses without having to insert a spraying catheter or use indigo carmine. The importance of simple methods and accurate diagnoses for clinical practice is indisputable. Thus, Li et al. showed that confocal laser endomicroscopy can be used to identify gastric superficial cancers with high validity and reliability. ${ }^{26}$ However, confocal laser endomicroscopy requires the intravenous administration of a contrast agent. In contrast, M-NBI can be utilized by simply pushing a button on the endoscope. In addition, evaluation of DLs and IMVPs is sufficient for diagnosis 
with M-NBI, whereas confocal laser endomicroscopy requires knowledge of histopathology procedures for diagnosis.

Major bleeding caused by an endoscopic biopsy is rarely reported. ${ }^{27}$ However, in our study, 2 patients experienced bleeding from the biopsy site. The best way of avoiding such bleeding is to avoid unnecessary biopsies. M-NBI, especially when used in conjunction with C-WLI, could help to reduce the number of unnecessary biopsies.

Our study has some limitations. First, the number of cancerous lesions was small, and it was less than the required sample size. This might be associated with insufficient power to evaluate sensitivity adequately. Then, further large numbers of patients for screening are needed to evaluate the sensitivity for diagnosing small gastric mucosal cancers of each modality. Second, this study was open-labeled because the endoscopists knew which imaging modality was in use. Thus, a blinded study was impossible. Third, there is no arm which includes dye-based imaging method such as indigocarmine or acetic acid. Indigo carmine and acetic acid are useful, but these dyes are only used in a few countries and institutes, and then, the standard worldwide endoscopic method to diagnose early gastric cancer is still C-WLI without any dye use. In addition, if we used a chromoendoscopy arm in this study, the required sample size would need to be enlarged and the study design and statistical analyses would be excessively complex. For these reasons, we did not include the dye-based imaging method.

Early detection of small gastric cancers makes it possible to effect a cure using minimally invasive treatments such as EMR and ESD. In this study, all of the newly diagnosed small gastric cancers were good candidates for these procedures. Among the 37 cancers removed, 35 (95\%) were mucosal. Early diagnosis using M-NBI and minimally invasive treatment is ideal for patients with gastric cancers, as it will improve their survival and quality of life. Although eradication of Helicobacter pylori is effective in reducing the 
incidence of gastric cancer, ${ }^{17,28}$ endoscopic examination using M-NBI in conjunction with CWLI should be indicated for high-incidence areas such as East Asia, South America, Eastern European countries, and Russia. ${ }^{29}$

\section{Gastric NBI study Investigators in Japan}

Noriya Uedo, Yoji Takeuchi (Osaka Medical Cancer for Cancer and Cardiovascular Diseases, Osaka), Hisashi Doyama, Yoshibumi Kaneko, Kenichi Takemura, Kazuhiro Miwa, Shinya Yamada (Ishikawa Prefectural Central Hospital, Ishikawa), Yutaka Saito, Ichiro Oda, Shigetaka Yoshinaga, Satoru Nonaka, Shusei Fukunaga (National Cancer Center Hospital, Tokyo), Manabu Muto, Yasumasa Ezoe, Shuko Morita, Takahiro Horimatsu (Kyoto University, Kyoto), Kenshi Yao, Takashi Nagahama, Hiroshi Tanabe, Takahiro Beppu, Yoichiro Ono, Masao Takeichi (Fukuoka University Chikushi Hospital, Fukuoka), Kazuhiro Kaneko, Tomonori Yano, Hiroaki Kon, Shinya Tsuruta (National Cancer Center Hospital East, Chiba), Yoshiro Kawahara, Toshio Uraoka, Seiji Kawano, Keisuke Hori (Okayama University Hospital, Okayama), Chizu Yokoi, Naoyoshi Nagata (National Center for Global Health and Medicine, Tokyo), Yasushi Sugiura (Kitano Hospital, Osaka), Hideki Ishikawa (Kyoto Prefectural University of Medicine, Kyoto), Tomoko Aoyama (Medical Research Support, Osaka). 


\section{Figure Legends}

Figure 1. Representative endoscopic findings for gastric small depressed lesions.

Panels $A-C$ show cancers and panels $D-F$ show noncancerous lesions. Panel A shows an endoscopic image obtained using conventional white-light imaging (C-WLI). A small depressed lesion (arrowheads) is evident in the anterior wall of the lower part of the gastric body. This lesion was evaluated as having an irregular margin (IM) and a spiny depressed area (SDA). Panel B shows an endoscopic image obtained using magnifying narrowband imaging (M-NBI), which enabled clear visualization of the demarcation line (DL) and an irregular microvascular pattern (IMVP). Panels $A^{\prime}$ and $B^{\prime}$ are schematic representations of the images shown in panels $A$ and $B$, respectively. Panel $C$ shows a lesion that was histologically diagnosed as a differentiated adenocarcinoma, Vienna Classification C4. Panel D shows an image obtained using C-WLI. A small reddish area (arrowheads) is evident in the anterior wall of the upper part of the gastric body. Because the depressed area was not "spiny" and because a definite margin was not apparent, this case was evaluated as not having an SDA or an IM. Panel E shows an image obtained using M-NBI, which enabled clear visualization of a DL and the absence of an IMVP. Panels $\mathrm{D}^{\prime}$ and $\mathrm{E}^{\prime}$ are schematic representations of the images shown in panels $D$ and $E$, respectively. Panel $F$ shows a lesion that was histologically diagnosed as gastritis, Vienna Classification C1.

Figure 2. Patient enrollment, randomization, and examination.

Figure 3. Positive predictive value (PPV) and negative predictive value (NPV) in each examination.

*The PPV for M-NBI was significantly higher than for C-WLI $(P=.001)$. The NPV in M-NBI was higher than that of $\mathrm{C}-\mathrm{WLI}$; however, the difference was not significant $(P=.16) .{ }^{*}$ Both 
PPV and NPV were significantly enhanced by additional examination using M-NBI compared with $\mathrm{C}-\mathrm{WLI}$ alone $(P<.001)$. 


\section{References}

1. Ferlay J, Shin HR, Bray F, et al. Estimates of worldwide burden of cancer in 2008: GLOBOCAN 2008. Int J Cancer 2010;127:2893-2917.

2. Tada M, Murakami A, Karita M, et al. Endoscopic resection of early gastric cancer. Endoscopy 1993;25:445-451.

3. Ono H, Kondo H, Gotoda $\mathrm{T}$, et al. Endoscopic mucosal resection for treatment of early gastric cancer. Gut 2001;48:225-229.

4. Gotoda T, Yanagisawa A, Sasako M, et al. Incidence of lymph node metastasis from early gastric cancer: estimation with a large number of cases at two large centers. Gastric Cancer 2000;3:219-225.

5. Everett SM, Axon AT. Early gastric cancer in Europe. Gut 1997;41:142-150.

6. Hirasawa T, Gotoda T, Miyata S, et al. Incidence of lymph node metastasis and the feasibility of endoscopic resection for undifferentiated-type early gastric cancer. Gastric Cancer 2009;12:148-152.

7. Tajiri H, Ohtsu A, Boku N, et al. Routine endoscopy using electronic endoscopes for gastric cancer diagnosis: retrospective study of inconsistencies between endoscopic and biopsy diagnoses. Cancer Detect Prev 2001;25:166-173.

8. Nakayoshi T, Tajiri H, Matsuda K, et al. Magnifying endoscopy combined with narrow band imaging system for early gastric cancer: correlation of vascular pattern with histopathology (including video). Endoscopy 2004;36:1080-1084.

9. Yao K, Iwashita A, Tanabe H, et al. Novel zoom endoscopy technique for diagnosis of small flat gastric cancer: a prospective, blind study. Clin Gastroenterol Hepatol 2007;5:869-878.

10. Ezoe $\mathrm{Y}$, Muto M, Horimatsu T, et al. Magnifying narrow-band imaging versus magnifying white-light imaging for the differential diagnosis of gastric small depressive lesions: a prospective study. Gastrointest Endosc 2010;71:477-484.

11. Yao K, Iwashita A, Tanabe H, et al. White opaque substance within superficial elevated gastric neoplasia as visualized by magnification endoscopy with narrow-band imaging: a 
new optical sign for differentiating between adenoma and carcinoma. Gastrointest Endosc 2008;68:574-580.

12. Kaise M, Kato M, Urashima M, et al. Magnifying endoscopy combined with narrow-band imaging for differential diagnosis of superficial depressed gastric lesions. Endoscopy 2009;41:310-315.

13. Kato M, Kaise M, Yonezawa J, et al. Magnifying endoscopy with narrow-band imaging achieves superior accuracy in the differential diagnosis of superficial gastric lesions identified with white-light endoscopy: a prospective study. Gastrointest Endosc 2010;72:523-529.

14. Tamai N, Kaise M, Nakayoshi T, et al. Clinical and endoscopic characterization of depressed gastric adenoma. Endoscopy 2006;38:391-394.

15. Uedo $\mathrm{N}$, Ishihara $\mathrm{R}$, lishi $\mathrm{H}$, et al. A new method of diagnosing gastric intestinal metaplasia: narrow-band imaging with magnifying endoscopy. Endoscopy 2006;38:819-824.

16. Bossuyt PM, Reitsma JB, Bruns DE, et al. The STARD statement for reporting studies of diagnostic accuracy: explanation and elaboration. Ann Intern Med 2003;138:W1-12.

17. Aoi T, Marusawa H, Sato T, et al. Risk of subsequent development of gastric cancer in patients with previous gastric epithelial neoplasia. Gut 2006;55:588-589.

18. Fukase K, Kato M, Kikuchi S, et al. Effect of eradication of Helicobacter pylori on incidence of metachronous gastric carcinoma after endoscopic resection of early gastric cancer: an open-label, randomised controlled trial. Lancet 2008;372:392-397.

19. Nakajima T, Oda I, Gotoda T, et al. Metachronous gastric cancers after endoscopic resection: how effective is annual endoscopic surveillance? Gastric Cancer 2006;9:93-98.

20. Gono K, Yamazaki K, Doguchi N, et al. Endoscopic observation of tissue by narrow band illumination. Opt Rev 2003;10:211-215.

21. Gono K, Obi T, Yamaguchi M, et al. Appearance of enhanced tissue feature in narrow-band endoscopic imaging. J Biomed Opt 2004;9:568-577.

22. Muto M, Katada C, Sano Y, et al. Narrow band imaging: a new diagnostic approach to visualize angiogenesis in the superficial neoplasia. Clin Gastroenterol Hepatol 2005;3(Suppl 1):S16-S20. 
23. Yao K, Nagahama T, So S, et al. Morphological correlation between ordinary and magnifying endoscopic findings with regard to small depressed-type gastric cancers. Stomach and Intestine 2006;41:781-794 [Article in Japanese].

24. Yao K, Oishi T, Matsui T, et al. Novel magnified endoscopic findings of microvascular architecture in intramucosal gastric cancer. Gastrointest Endosc 2002;56:279-284.

25. Schlemper RJ, Riddell RH, Kato Y, et al. The Vienna classification of gastrointestinal epithelial neoplasia. Gut 2000;47:251-255.

26. Li WB, Zuo XL, Li CQ, et al. Diagnostic value of confocal laser endomicroscopy for gastric superficial cancerous lesions. Gut 2011;60:299-306.

27. Sieg A, Hachmoeller-Eisenbach U, Eisenbach T. Prospective evaluation of complications in outpatient Gl endoscopy: a survey among German gastroenterologists. Gastrointest Endosc 2001;53:620-627.

28. Chiba T, Marusawa $\mathrm{H}$, Seno $\mathrm{H}$, et al. Mechanism for gastric cancer development by Helicobacter pylori infection. J Gastroenterol Hepatol 2008;23:1175-1181.

29. Jemal A, Center MM, DeSantis C, et al. Global patterns of cancer incidence and mortality rates and trends. Cancer Epidemiol Biomarkers Prev 2010;19:1893-1907. 
Table 1. Baseline Characteristics of the Study Participants according to Treatment Group

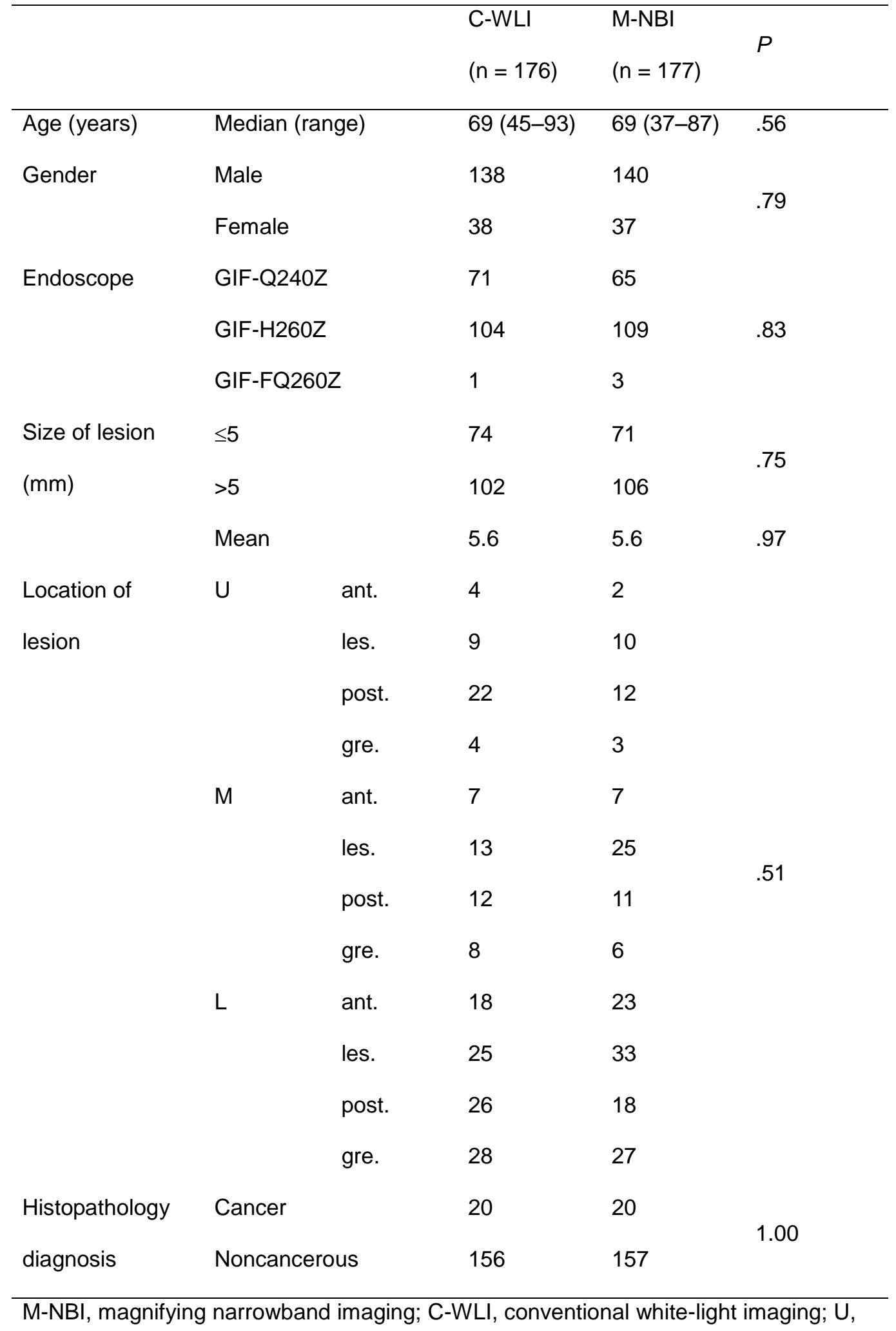


upper third; M, middle third; L, lower third; ant., anterior wall; les., lesser curvature;

post., posterior; gre., greater curvature. 
Table 2. Endoscopic Diagnoses for All Small Depressed Lesions

\begin{tabular}{|c|c|c|c|c|c|c|c|}
\hline \multirow[b]{2}{*}{ Group } & \multirow[b]{2}{*}{ Method } & \multicolumn{3}{|c|}{ Cancerous lesion } & \multicolumn{3}{|c|}{ Noncancerous lesion } \\
\hline & & $\begin{array}{l}\text { Correct } \\
\text { diagnosis }\end{array}$ & $\begin{array}{l}\text { Incorrect } \\
\text { diagnosis }\end{array}$ & $\begin{array}{l}\text { Inconclusive } \\
\text { diagnosis }\end{array}$ & $\begin{array}{l}\text { Correct } \\
\text { diagnosis }\end{array}$ & $\begin{array}{l}\text { Incorrect } \\
\text { diagnosis }\end{array}$ & $\begin{array}{l}\text { Inconclusive } \\
\text { diagnosis }\end{array}$ \\
\hline $\mathrm{M}-\mathrm{NBI}$ & $\mathrm{M}-\mathrm{NBI}$ & $\begin{array}{l}12 / 20 \\
(60.0 \%)\end{array}$ & $\begin{array}{l}7 / 20 \\
(35.0 \%)\end{array}$ & $\begin{array}{l}1 / 20 \\
(5.0 \%)\end{array}$ & $\begin{array}{l}146 / 157 \\
(93.0 \%)\end{array}$ & $\begin{array}{l}9 / 157 \\
(5.7 \%)\end{array}$ & $\begin{array}{l}2 / 157 \\
(1.3 \%)\end{array}$ \\
\hline C-WLI & C-WLI & $\begin{array}{l}8 / 20 \\
(40.0 \%)\end{array}$ & $\begin{array}{l}12 / 20 \\
(60.0 \%)\end{array}$ & $\begin{array}{l}0 / 20 \\
(0 \%)\end{array}$ & $\begin{array}{l}100 / 156 \\
(64.1 \%)\end{array}$ & $\begin{array}{l}50 / 156 \\
(32.1 \%)\end{array}$ & $\begin{array}{l}6 / 156 \\
(3.8 \%)\end{array}$ \\
\hline & $\begin{array}{l}\text { C-WLI } \\
+\mathrm{M}-\mathrm{NBI}\end{array}$ & $\begin{array}{l}19 / 20 \\
(95.0 \%)\end{array}$ & $\begin{array}{l}1 / 20 \\
(5.0 \%)\end{array}$ & $\begin{array}{l}0 / 20 \\
(0 \%)\end{array}$ & $\begin{array}{l}149 / 156 \\
(95.5 \%)\end{array}$ & $\begin{array}{l}5 / 156 \\
(3.2 \%)\end{array}$ & $\begin{array}{l}2 / 156 \\
(1.3 \%)\end{array}$ \\
\hline
\end{tabular}

$\mathrm{M}-\mathrm{NBI}$, magnifying narrowband imaging; C-WLI, conventional white-light imaging. 
Table 3. Diagnostic Performance of C-WLI and M-NBI for Gastric Small Depressed Lesions

\begin{tabular}{|c|c|c|c|c|c|}
\hline Group & Method & Accuracy & Sensitivity & Specificity & $\begin{array}{l}\text { Exam. time } \\
\text { (s) } \\
\text { Median } \\
\text { (IQR) }\end{array}$ \\
\hline $\mathrm{M}-\mathrm{NBI}$ & $\mathrm{M}-\mathrm{NBI}$ & $\begin{array}{l}90.4^{*} \\
(85.1-94.3)\end{array}$ & $\begin{array}{l}60.0 \\
(36.1-80.9]\end{array}$ & $\begin{array}{l}94.3^{*} \\
(89.4-97.3)\end{array}$ & $\begin{array}{l}55^{*} \\
(23-97)\end{array}$ \\
\hline C-WLI & C-WLI & $\begin{array}{l}64.8 \\
(57.2-71.8)\end{array}$ & $\begin{array}{l}40.0 \\
(19.1-63.9)\end{array}$ & $\begin{array}{l}67.9 \\
(60.0-75.2)\end{array}$ & $\begin{array}{l}21 \\
(12-40)\end{array}$ \\
\hline & $\begin{array}{l}\mathrm{C}-\mathrm{WLI}+\mathrm{M}- \\
\mathrm{NBI}\end{array}$ & $\begin{array}{l}96.6^{* *} \\
(93.5-99.1)\end{array}$ & $\begin{array}{l}95.0^{* *} \\
(75.1-99.9)\end{array}$ & $\begin{array}{l}96.8^{* *} \\
(92.7-99.0)\end{array}$ & $\begin{array}{l}72^{* *} \\
(40-144)\end{array}$ \\
\hline
\end{tabular}

${ }^{\star} P<.001$ for M-NBI vs C-WLI; ${ }^{*} P<.001$ for C-WLI vs C-WLI + M-NBI

M-NBI, magnifying narrowband imaging; C-WLI, conventional white-light imaging;

exam. time, examination time; $\mathrm{Cl}$, confidence interval; IQR, interquartile range. 
Table 4. Clinical Course and Pathological Diagnosis of Patients With Gastric Cancers

\begin{tabular}{lll}
\hline No. of patients & & 40 \\
Treatment & EMR/ESD & $2 / 30$ \\
& Surgery & 5 \\
& No treatment & 3 \\
Histological type & Adenocarcinoma & 40 \\
& (intestinal type) & $(39)$ \\
& (diffuse type) & $(1)$ \\
& Other diagnosis & 0 \\
Pathological depth & $\mathrm{m}$ & 35 \\
& sm & 2 \\
& mp $\leq$ & 0 \\
& Unknown & 3 \\
\hline EMR5, endoscopic mucosal resection; ESD, endoscopic \\
submucosal dissection; \\
muscularis propria.
\end{tabular}


Click here to download high resolution image

Figure 1

\section{Cancer}
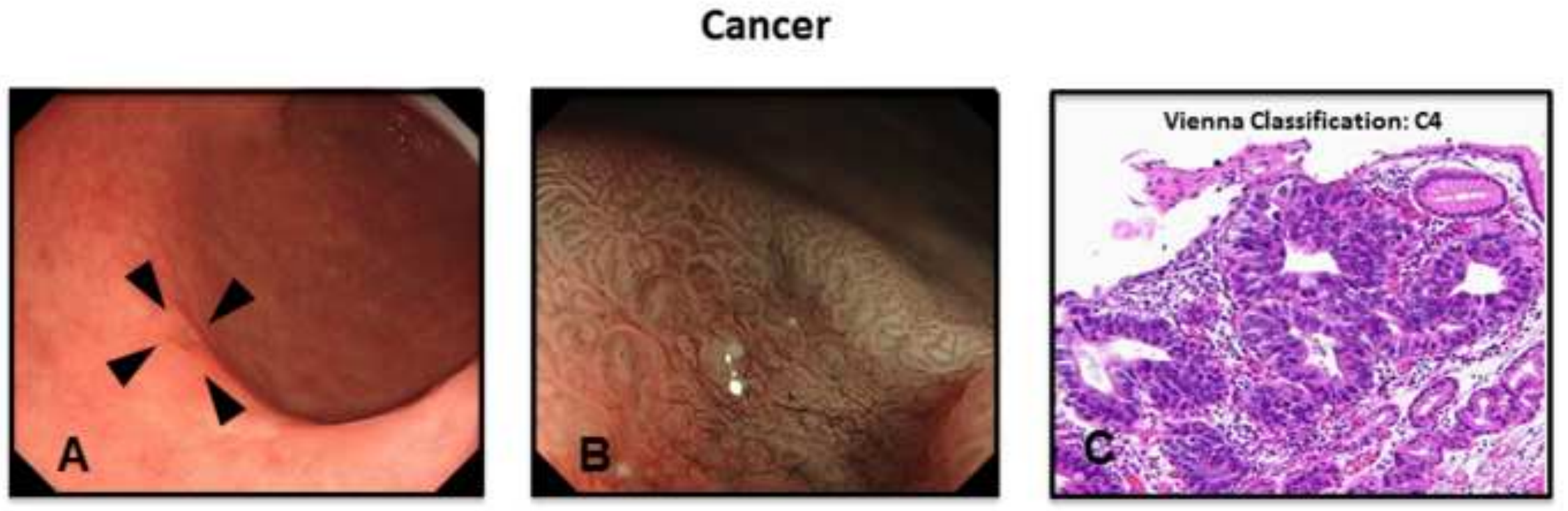

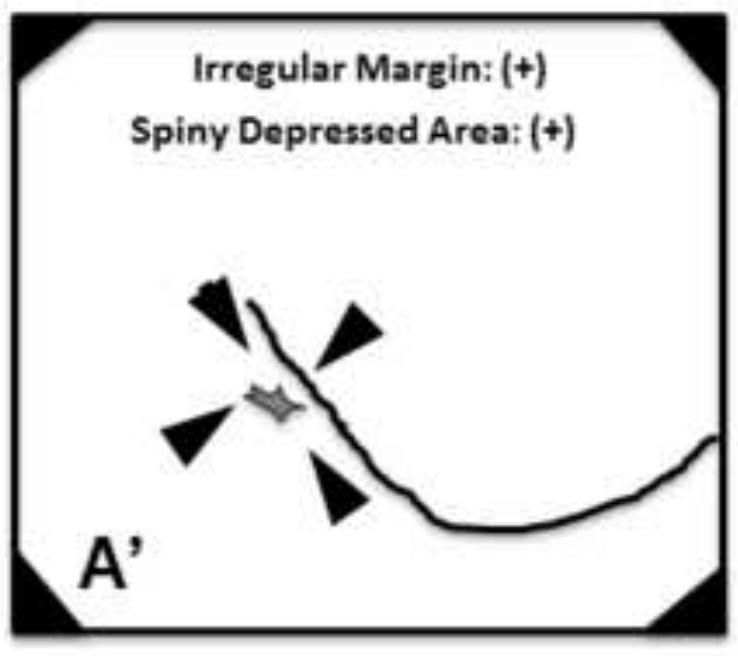

\section{Irregularmargin: $(+)$} piny Depressed Area: (+)

$\mathbf{A}^{\prime}$

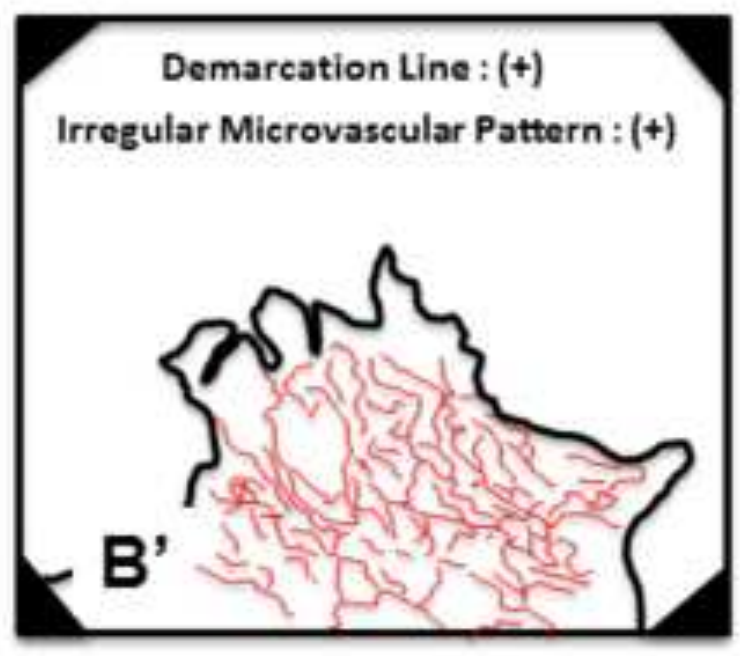

Irregular Microvascular Pattern : (+) 
Figure 1 cont'

\section{Noncancerous Lesion}
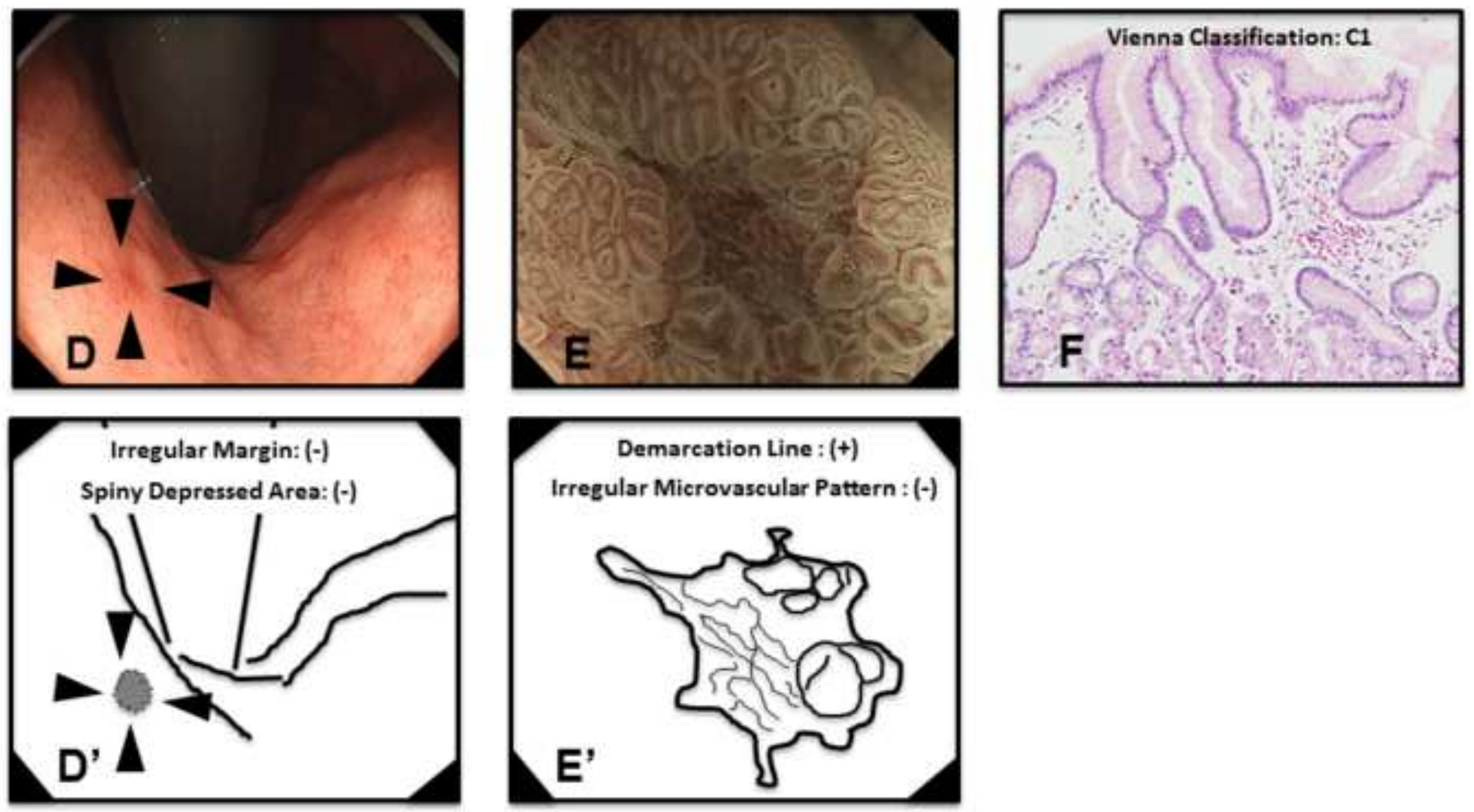


\section{Figure 2}

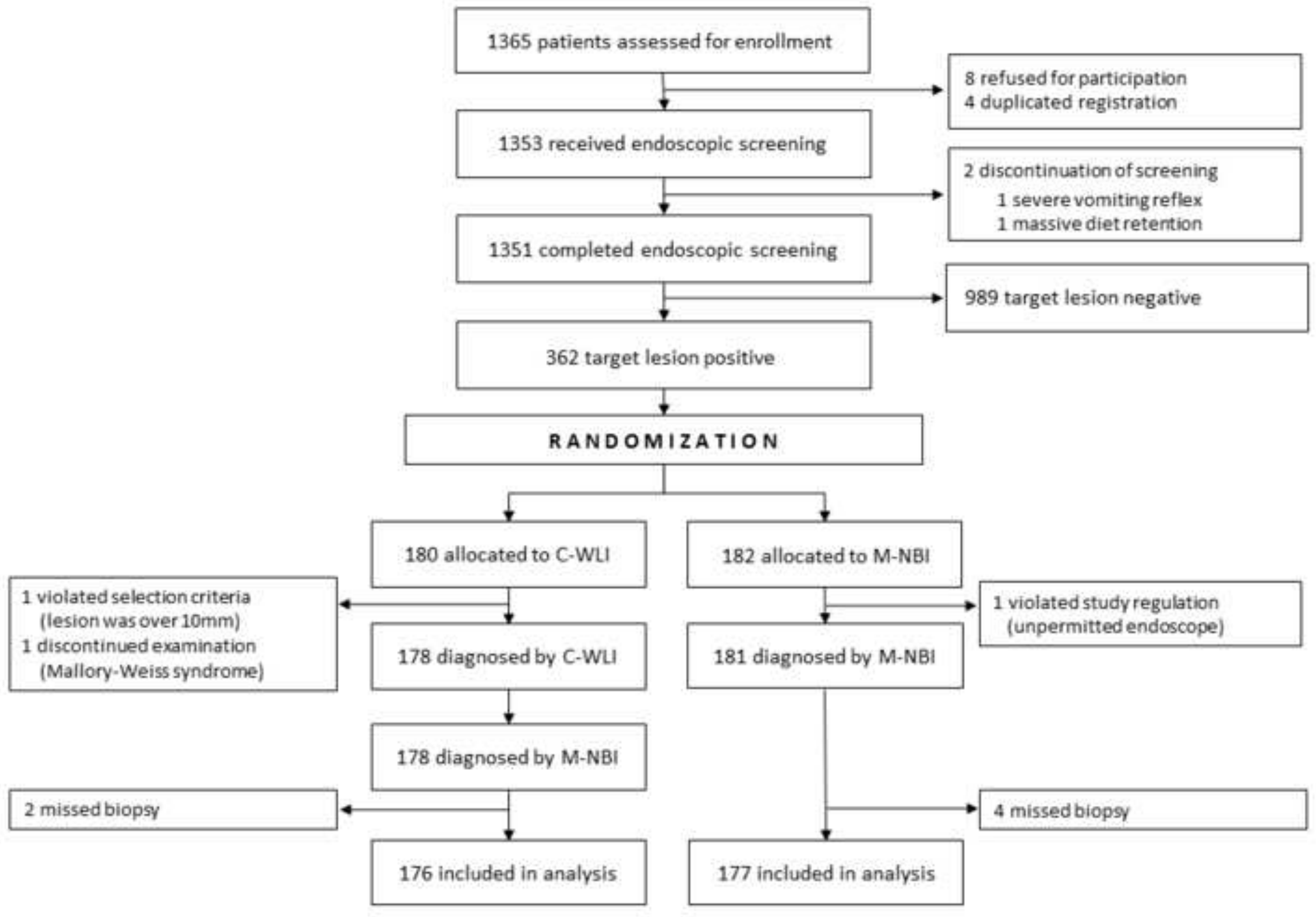


Click here to download high resolution image

\section{Figure 3}

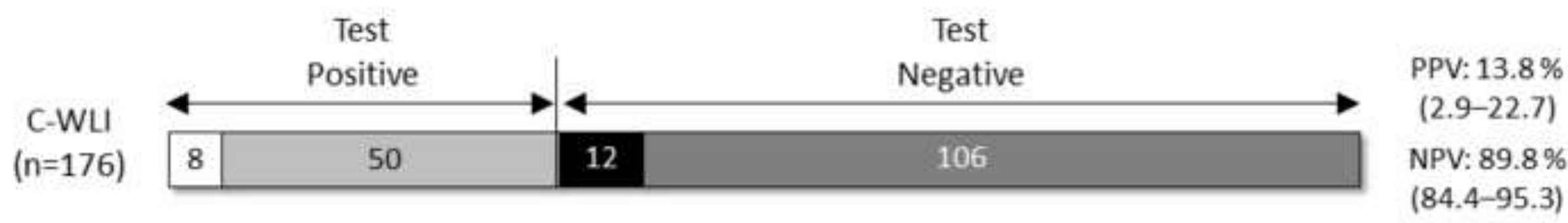

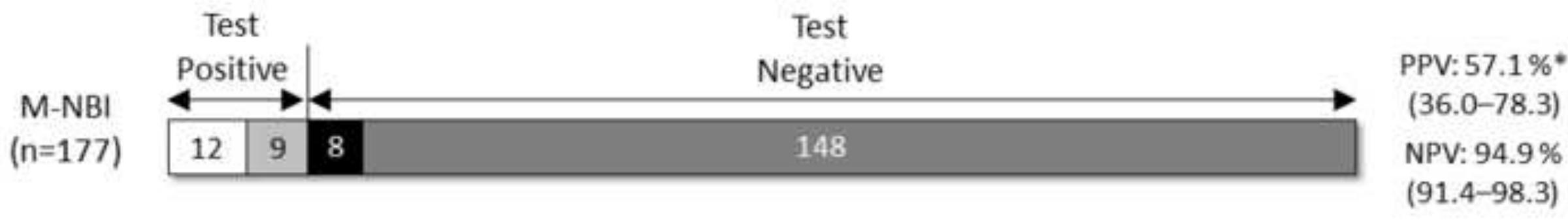

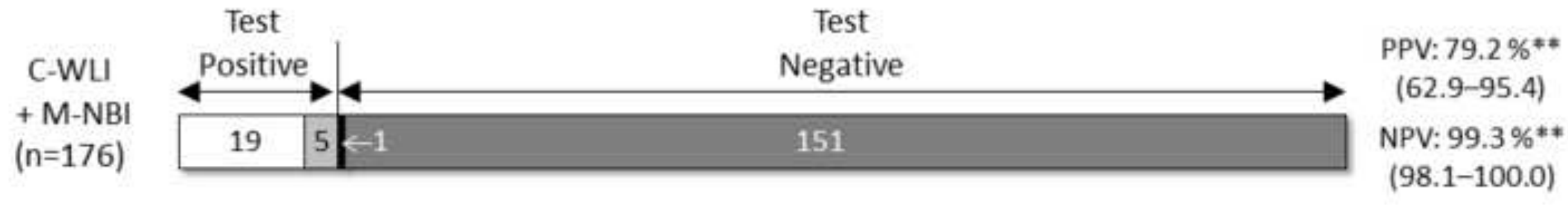

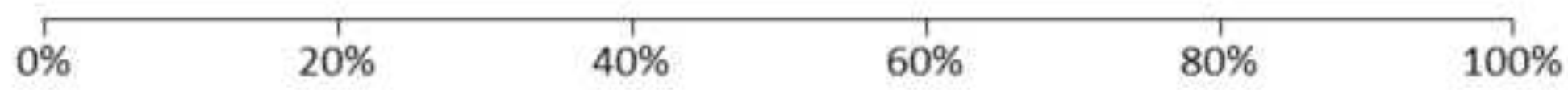

True Positive $\square$ False Positive $\square$ False Negative $\square$ True Negative
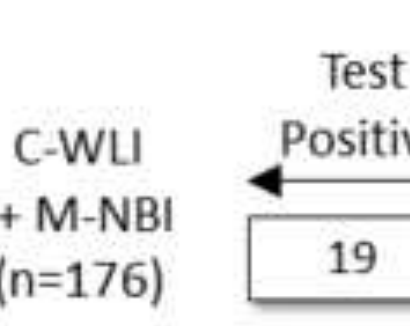

$$
\text { False Positive }
$$

$$
\text { True Negative }
$$

P. $13.8 \%$ NPV: $89.8 \%$ (84.4-95.3) 


\section{CONSORT 2010 checklist of information to include when reporting a randomised trial*}

$\begin{array}{lccc} & \text { Item } & & \text { Reported } \\ \text { Section/Topic } & \text { No Checklist item } & \text { on page No }\end{array}$

Title and abstract

1a Identification as a randomised trial in the title

1b Structured summary of trial design, methods, results, and conclusions (for specific guidance see CONSORT for abstracts)

\section{Introduction}

Background and

objectives

\section{Methods}

Trial design

Participants

Interventions

Outcomes

Sample size

Randomisation:

Sequence generation
Scientific background and explanation of rationale

Specific objectives or hypotheses

$3 a$

$3 b$

$4 \mathrm{a}$

$4 b$

5

The interventions for each group with sufficient details to allow replication, including how and when they were actually administered

6a Completely defined pre-specified primary and secondary outcome measures, including how and when they were assessed

$6 \mathrm{~b}$ Any changes to trial outcomes after the trial commenced, with reasons

7a How sample size was determined

$7 \mathrm{~b}$ When applicable, explanation of any interim analyses and stopping guidelines

8a Method used to generate the random allocation sequence

$8 \mathrm{~b}$ Type of randomisation; details of any restriction (such as blocking and block size)

on page No

No.

Because the title exceeds the journal limit of 120 characters with spaces. \#4

$$
\text { \#5-6 }
$$$$
\text { \#5-6 }
$$

\begin{tabular}{l} 
\#6-7 \\
\hline No change \\
\hline$\# 9$ \\
\hline$\# 7-8$ \\
\hline$\# 8-10$ \\
\hline No change \\
\hline$\# 10$ \\
\hline No setting \\
$\# 7$ \\
\hline$\# 7$
\end{tabular}


Allocation concealment mechanism

Implementation

Blinding

Statistical methods

\section{Results}

Participant flow (a diagram is strongly recommended)

Recruitment

Baseline data

Numbers analysed

Outcomes and estimation

Ancillary analyses

Harms

\section{Discussion}

Limitations

Generalisability

Interpretation
9 Mechanism used to implement the random allocation sequence (such as sequentially numbered containers), describing any steps taken to conceal the sequence until interventions were assigned

10 Who generated the random allocation sequence, who enrolled participants, and who assigned participants to interventions

11a If done, who was blinded after assignment to interventions (for example, participants, care providers, those assessing outcomes) and how

$11 \mathrm{~b}$ If relevant, description of the similarity of interventions

12a Statistical methods used to compare groups for primary and secondary outcomes

12b Methods for additional analyses, such as subgroup analyses and adjusted analyses

13a For each group, the numbers of participants who were randomly assigned, received intended treatment, and were analysed for the primary outcome

13b For each group, losses and exclusions after randomisation, together with reasons

14a Dates defining the periods of recruitment and follow-up

14b Why the trial ended or was stopped

15 A table showing baseline demographic and clinical characteristics for each group

16 For each group, number of participants (denominator) included in each analysis and whether the analysis was by original assigned groups

17a For each primary and secondary outcome, results for each group, and the estimated effect size and its precision (such as $95 \%$ confidence interval)

17b For binary outcomes, presentation of both absolute and relative effect sizes is recommended

18 Results of any other analyses performed, including subgroup analyses and adjusted analyses, distinguishing pre-specified from exploratory

19 All important harms or unintended effects in each group (for specific guidance see CONSORT for harms)

20 Trial limitations, addressing sources of potential bias, imprecision, and, if relevant, multiplicity of analyses

21 Generalisability (external validity, applicability) of the trial findings

22 Interpretation consistent with results, balancing benefits and harms, and considering other relevant evidence

\section{\#7}

Open labelled

\begin{tabular}{l}
$\# 10-11$ \\
\hline $10-11$ \\
\hline
\end{tabular}

$\# 11-12$

\section{$\# 11$ and}

figure 2

$\# 11$

$(-)$

table 1

\#11-12 and

figure 2

$\# 12-13$

$$
\text { \#12-13 }
$$

\#12-13

\#13

\#16

\#13-14

\#16-17

\#16-17

\section{Other information}




$\begin{array}{lll}\text { Registration } & 23 & \text { Registration number and name of trial registry } \\ \text { Protocol } & 24 & \text { Where the full trial protocol can be accessed, if available }\end{array}$

\#4, \#6 Full trial protocol is submitted as one of the supplement Funding

files.

\#2

*We strongly recommend reading this statement in conjunction with the CONSORT 2010 Explanation and Elaboration for important clarifications on all the items. If relevant, we also recommend reading CONSORT extensions for cluster randomised trials, non-inferiority and equivalence trials, non-pharmacological treatments, herbal interventions, and pragmatic trials. Additional extensions are forthcoming: for those and for up to date references relevant to this checklist, see www.consort-statement.org. 


\section{Supplementary Materials and Methods}

\section{Study flow}

Written informed consent was obtained from all eligible patients. To detect a target lesion, endoscopic screening was done using conventional white-light imaging (C-WLI). If no target lesion was detected, routine endoscopic examination were performed without study entry. When a target lesion was detected, patients were immediately assigned randomly to undergo detailed examination using C-WLI or magnifying narrowband imaging (M-NBI). For the C-WLI group, M-NBI examination was performed after completion of a diagnosis based on C-WLI. After all diagnoses were compiled, at least one biopsy specimen was obtained from the target lesion. The primary aim of this study was to compare directly the real-time diagnostic accuracy of two randomly assigned endoscopic modalities; C-WLI and M-NBI (solid line box). The secondary aim of this study was to evaluate the additional effect of performing M-NBI after C-WLI (dash line box).

\section{Diagnostic method based on endoscopic findings}

Endoscopic diagnoses were made according to the combination of the endoscopic findings. In the case of conventional white-light imaging (C-WLI), an irregular margin and a spiny depressed area were used for the diagnostic findings. In the case of magnifying narrowband imaging (M-NBI), a demarcation line between the depressed cancerous lesion and the surrounding noncancerous area and an irregular microvascular pattern inside the lesion were used for the diagnosis. If both findings were present in each examination, the diagnosis of "cancer" was made. If either finding was indeterminate, the diagnosis was "inconclusive." If either or both findings were absent, the diagnosis was "noncancerous".

Supplementary figure1: Study flow

Supplementary figure 2: Diagnostic method based on endoscopic findings 


\section{Supplement 1}

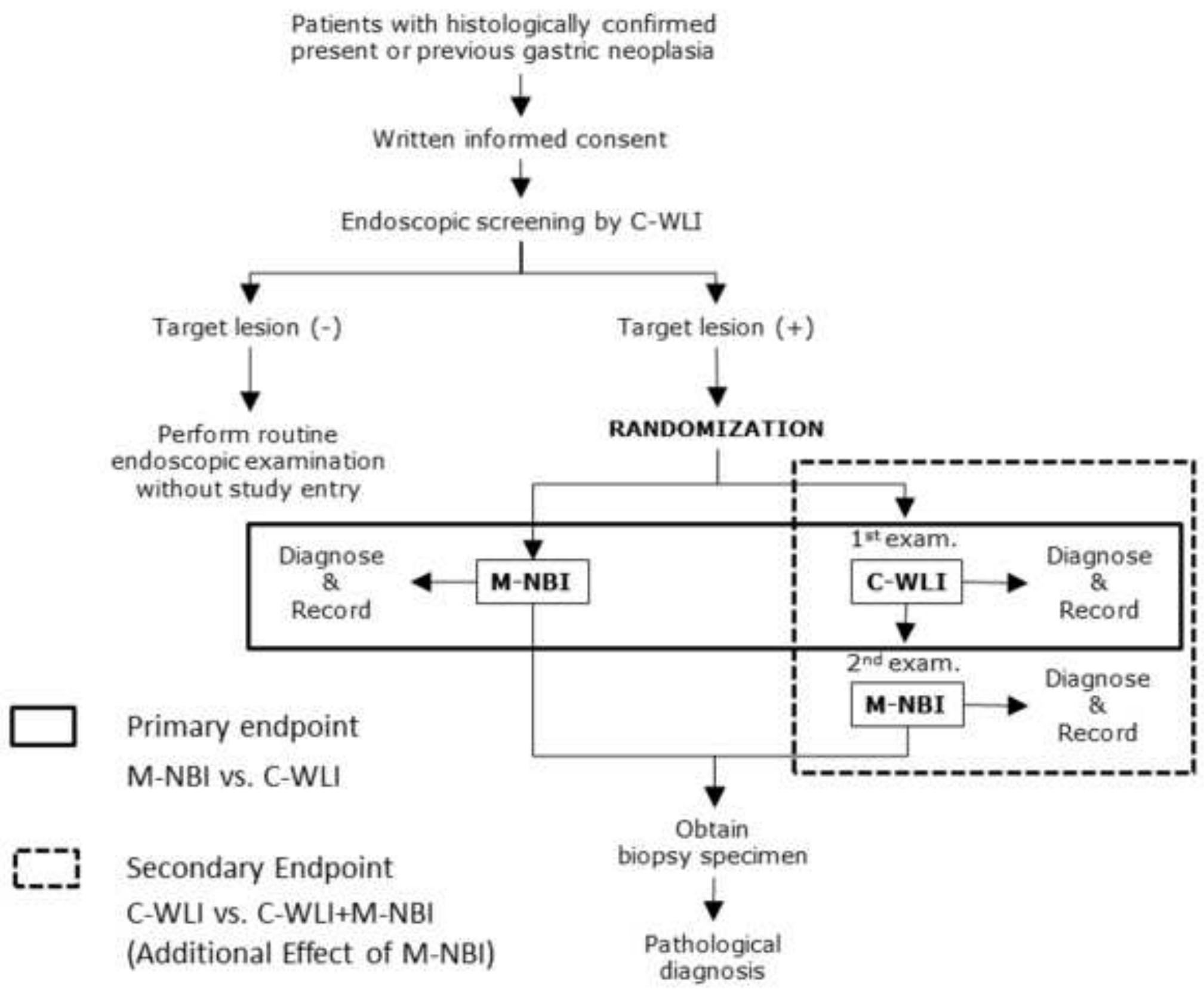

present or previous gastric neoplasia

Written informed consent

doscopic screening by C-WL

Target lesion (-)

Perform routine doscopic examination without study entry

M-NBI vs. C-WLI

(Additional Effect of M-NBI)

diagnosis

biopsy specimen

Pathological

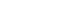


Supplement 2

\section{Conventional White-light Imaging (C-WLI)}

\begin{tabular}{|c|c|c|c|c|}
\hline & & \multicolumn{3}{|c|}{ Spiny Depressed Area } \\
\hline & & present & absent & indeterminate \\
\hline \multirow{3}{*}{$\begin{array}{c}\text { Irregular } \\
\text { Margin }\end{array}$} & present & cancer & \multirow[b]{2}{*}{ noncancerous } & \multirow[b]{3}{*}{ inconclusive } \\
\hline & absent & & & \\
\hline & indeterminate & & & \\
\hline
\end{tabular}


Supplement 2 cont'

Magnifying Narrowband Imaging (M-NBI)

\begin{tabular}{|c|c|c|c|c|}
\hline & & \multicolumn{3}{|c|}{ Irregular Microvascular Pattern } \\
\hline & & present & absent & indeterminate \\
\hline \multirow{3}{*}{$\begin{array}{l}\text { Demarcation } \\
\text { Line }\end{array}$} & present & cancer & \multirow[b]{2}{*}{ noncancerous } & \multirow[b]{3}{*}{ inconclusive } \\
\hline & absent & & & \\
\hline & indeterminate & & & \\
\hline
\end{tabular}

